\title{
IDENTIFICATION AND MANAGEMENT OF DIFFICULT STROKE AND TIA SYNDROMES
} sually, difficulty in the diagnosis and management of stroke occurs because of the rarity of the presentation, ignorance about the condition, or the lack of scientific clinical trial data to support clinical decisions. This article focuses on a very individual selection of topics, with much of the advice concerning management representing a personal view. It is not intended to be comprehensive, and is limited mainly to transient ischaemic attack (TIA) and ischaemic stroke.

The importance of retaking a detailed history from the patient and their carers or friends in difficult cases cannot be overemphasised. Radiological investigations should be reviewed, taking the history into account, and remembering that minor vascular abnormalities are common in the elderly and that optimum sequences may not have been used, particular with magnetic resonance imaging (MRI). More extensive investigation may be required.

\section{DIFFERENTIAL DIAGNOSIS OF TRANSIENT SYMPTOMS}

The difficulty of diagnosing TIA is illustrated by the wide variety of diagnoses made in patients referred to vascular clinics (table 1). ${ }^{1}$ The sudden onset of neurological symptoms is a useful criterion for the diagnosis of TIA. Most recover within 10-30 minutes. As a general principle, the syndromes of TIA will mimic those of stroke, since the symptoms are a function of the vascular territory of the occluded artery or arteriole. Thus isolated dizziness or vertigo, or loss of consciousness, are unlikely to be TIAs, whereas pure unilateral motor weakness-provided it involves at least two of face, arm or leg-can be clearly recognised as a lacunar TIA. The symptoms of most TIAs are loss of function, such as aphasia, weakness or numbness, as opposed to positive symptoms such as tingling or involuntary movements, which are more likely to have a non-ischaemic cause.

Haemodynamic TIAs are an important exception. These are usually associated with very severe internal carotid artery stenosis $(>95 \%)$ or occlusion. ${ }^{2}$ A TIA that occurs with activities associated with relative hypotension (for example, orthostasis, a hot bath) is likely to be haemodynamic. The symptoms of haemodynamic transient monocular blindness (TMB) are characteristically described by the patient as a "white out" or as checkerboard distortion of vision similar to looking through frosted glass, in contrast to embolic TMB where the loss of vision is described as blackness or a curtain coming down over the site of one eye. Haemodynamic TMB may be precipitated by going from a dark room into bright sunlight or even by looking at a bright light. In these cases, fundal examination may show poor perfusion pressure on the retinal arterioles which can be seen to pulsate and collapse in diastole in response to a slight pressure on the eyeball. In severe cases, fundoscopy may also show the features of a low pressure ischaemic retinopathy, with dilated veins, peripheral microaneurysms, and blot haemorrhages.

The symptoms of haemodynamic hemisphere TIAs occasionally consist of jerking of the arm and can easily be confused with focal seizures from other causes. Haemodynamic TIAs are an indication for angiography to visualise the whole of the carotid artery; often the patient has extensive atheromatous disease with tandem stenoses of the carotid bifurcation and distal internal carotid artery (fig 1).

\section{Migraine}

The focal symptoms of migraine with aura precede the headache in most cases and usually last from 10-30 minutes; focal symptoms lasting more than an hour are unusual in migraine. The focal symptoms of migraine are fairly characteristic, namely visual fortification spectra, hemianopia or less commonly perioral tingling with tingling in one arm. Isolated dysphasia is the only other transient symptom that I am happy to accept as migraine aura, if followed by otherwise typical headache and accompanying symptoms; however, it is helpful to hear a history of a march from typical visual symptoms or tingling in one limb to isolated dysphasia (usually fairly mild), over 15 minutes or so. The difficulty arises when a patient presents with symptoms suggestive of migraine aura without headache. A prior history of more typical migraine headache is helpful, but one should be aware of attributing focal symptoms to migraine rather than TIA 
Table 1 Final diagnosis in 211 new patients referred to a cerebrovascular disease clinic.

\begin{tabular}{ll}
\hline Diagnosis & $\begin{array}{l}\text { Percentage of } \\
\text { patients }\end{array}$ \\
\hline Cerebrovascular disease & 71 \\
Cerebral infarct & 32 \\
TIA & 30 \\
Asymptomatic carotid disease & 6 \\
Vascular dementia & 2 \\
Primary intracerebral haemorrhage & 1 \\
Non-cerebrovascular disease & 29 \\
Cardiac syncope & 5 \\
Migraine & 5 \\
Vestibular dysfunction & 4 \\
Epilepsy & 2 \\
Transient global amnesia & 2 \\
Cerebral tumour & 1 \\
Tension headache & 1 \\
No diagnosis made & 4 \\
Other* & 5
\end{tabular}

*One each (0.5\%) of the following: multiple sclerosis, motor neurone disease, cerebellar degeneration, ischaemic retinopathy, radiculopathy, chorea, Alzheimer's disease, hypoglycaemia, anxiety.

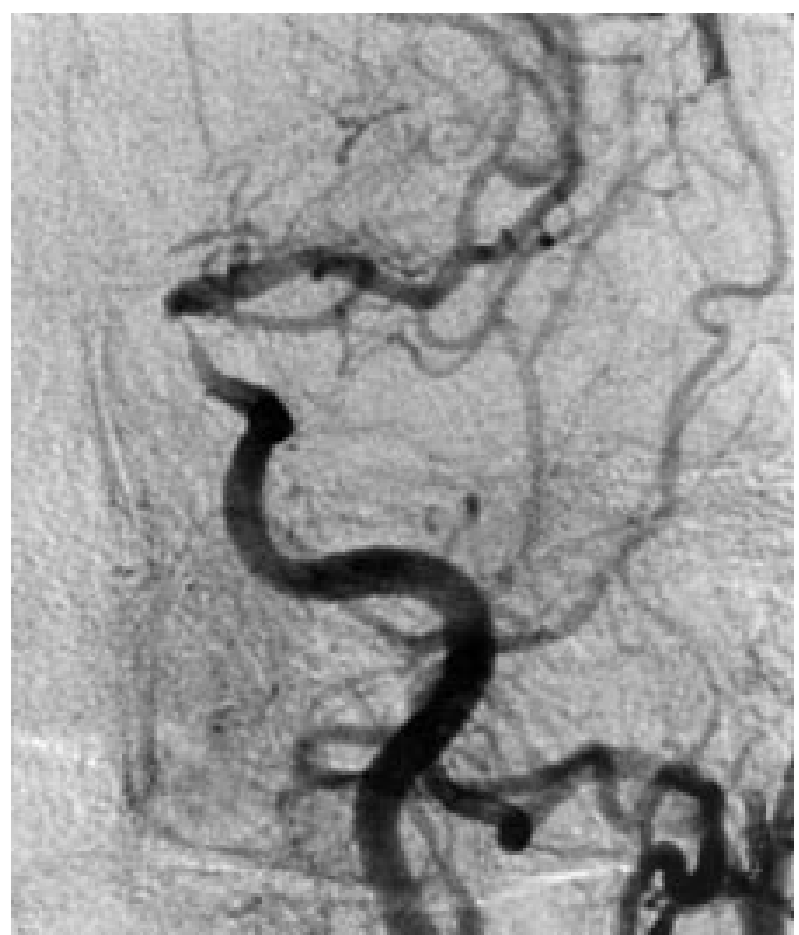

Figure 1 Digital subtraction angiogram showing a tight distal carotid stenosis in a diabetic man who presented with attacks of ipsilateral transient monocular "white out" of vision precipitated by bright light.

simply because the patient is a migraineur. I scan patients with focal symptoms without headache to exclude focal lesions, particularly arteriovenous malformations, except when there is a history of typical fortification spectra when I usually accept the diagnosis without further investigation. The diagnosis of migraineous infarction is discussed later.

\section{Migraine equivalent of middle age or late onset migraine accompaniment}

These synonymous terms describe the fairly common occurrence in middle aged patients of focal symptoms very similar, or identical, to those of the aura of migraine, without headache or a history of previous migraine. ${ }^{3}$ The patients may have coincidental vascular risk factors, but the occurrence of migraine equivalent does not appear to predict an increased risk of stroke, and patients can be reassured about the benign nature of the symptoms.

\section{Transient global amnesia}

Transient global amnesia is often confused with TIA. However, the disorder is distinct and does not carry the import of an increased risk of stroke. ${ }^{4}$ The attacks are often longer than TIAs, typically lasting 4-8 hours. Witnesses describe the patient as being confused, repeatedly asking the same questions, and being unable to recollect recent events. However, the patient often recognises family and the environment, if it has been familiar to him or her for some time. The patients are not dysphasic, which is a helpful distinguishing feature from a dominant hemisphere TIA, and they do not have any other focal features other than the amnesia. Bilateral thalamic ischaemia may cause an amnesic syndrome similar to transient global amnesia, but there will usually be other midbrain features.

\section{Progressive symptoms}

Classically, the symptoms of stroke are considered to be of sudden onset. However, a proportion of patients progress over the first day or two after onset. This is more common in patients with large vessel occlusion but is also seen in lacunar infarction. Cerebellar infarction and haemorrhage have a propensity to progress because of oedema and brain stem compression. In cerebellar, lateral medullary or brain stem infarction, progression may reflect propagation of thrombus in the vertebral artery to involve the basilar artery and its perforators. The patient may initially complain of vertigo or intermittent diploplia, which may be misinterpreted as a vestibular disturbance. These symptoms may improve or stabilise for several days or even a week, before the patient deteriorates with the development of progressive brain stem symptoms and signs, including coma. The history can be misinterpreted as indicating encephalitis, especially if the patient has headache or fever. Computed tomography (CT) may be normal in patients with posterior fossa ischaemia, and it is particularly important to consider the diagnosis and perform MRI before doing a lumbar puncture. Lumbar puncture in patients with significant cerebellar or brain stem oedema secondary to infarction can be hazardous and cause rapid deterioration.

\section{Malignant middle cerebral artery oedema}

An important cause of progressive symptoms in supratentorial infarction is malignant middle cerebral artery oedema. Cytotoxic oedema causes swelling of the hemisphere with brain stem compression in patients with infarction in most or all of the middle cerebral artery territory. Clinically, the patient presents with the classical symptoms of ischaemia in the middle cerebral artery territory, but initially remains alert. There is then a delay of $24-48$ hours before the onset of a rapid decline in conscious level leading to unresponsiveness and often death, sometimes within a few hours. The syndrome is usually seen in younger patients, because cerebral atrophy associated with aging protects against the rapid rise in intracranial pressure. Malignant cerebral oedema should be anticipated in young patients who have early signs of complete middle cerebral artery infarction on CT present within the first few hours of onset. The syndrome has a very poor prognosis when treated medically, but recent case series suggest that surgical decompressive craniectomy improves outcome in selected patients. ${ }^{5}$ The likelihood of severe residual aphasia argues against considering craniectomy in patients with dominant hemisphere infarction, unless the dysphasia is mild. 


\section{Summary 1}

- The symptoms of haemodynamic TIA include white out of vision and jerking of one arm

- Cerebellar infarction and haemorrhage have a particular tendency to progress

- Malignant middle cerebral artery oedema is an indication for considering craniectomy

- Heparin is not beneficial in acute stroke, even in patients with progressive symptoms

- Additional investigations are warranted in younger patients with stroke, but no cause may be found in as many as $40 \%$

- Migraine-like headache caused by stroke or carotid dissection is more common than stroke caused by migraine

Although it is tempting to heparinise patients with progressive stroke, two relatively small randomised trials showed no evidence that intravenous heparin in this situation is beneficial ${ }^{6}{ }^{7}$; the large randomised trials of subcutaneous heparin in stroke of any type also showed no overall benefit to anticoagulation. ${ }^{8}$

\section{The young adult with ischaemic stroke}

In clinical practice most young adults with stroke do not have a rare underlying disorder responsible for their symptoms, and the cause will often be suggested by the previous history or examination - for example, hypertension or heart disease. However, it is reasonable to investigate all patients under a certain age (for example, 50 years old) more extensively (see box right). In as many as $27 \%$ of cases of young stroke, no cause is apparent, ${ }^{9}$ although many have risk factors for stroke (for example, smoking) of uncertain relevance. In patients with no obvious cause, recurrence is unusual and it seems likely that in many an unknown factor or combination of factors, such as a recent infection, has temporarily resulted in hypercoagulability or endothelial injury, which by chance has tipped the balance of coagulation and thrombolysis over the threshold of thrombosis.

\section{Carotid and vertebral artery dissection}

Carotid and vertebral artery dissection account for a significant proportion of cases $(5-10 \%)$ of young stroke, but is rare above the age of 50 years. A history of minor or major neck trauma, abnormal neck postures, or of joint hypermobility may be obtained. The diagnosis is suggested by pain in the neck before or at the time of stroke onset and finding of a Horner's syndrome with an anterior circulation syndrome. Carotid dissection may occasionally precipitate migraine-like symptoms, including visual aura. Aortic arch dissection should be considered in patients with Marfan's syndrome or pseudoxanthoma elasticum.

\section{Mitochondrial cytopathy}

Mitochondrial encephalomyopathy with lactic acidosis and stroke-like episodes (MELAS) is often considered in the differential diagnosis of young stroke. It is rare, however, and I do not routinely investigate for this condition unless the patient has other features to suggest mitochondrial diseasefor example, myopathy, encephalopathy, prolonged migraine-like headache with vomiting, seizures, ophthalmoplegia, or the CT or MRI has the characteristic appearances in the occipital lobes not confined to the territory of the posterior cerebral arteries. ${ }^{10}$

\section{Hemiplegic migraine}

One of the more common misdiagnoses in patients with stroke is the attribution of focal symptoms associated with throbbing headache in a teenager or young patient to migraine. For some reason, almost all trainees know of hemiplegic migraine. However, the condition is extremely rare. Hemiplegic migraine should be regarded as a separate syndrome to migraine with aura. The hemiplegia accompanies or follows the onset of severe headache, may alternate from side to side within or between attacks, can be associated with fever, drowsiness, confusion or coma, and may last for several days. ${ }^{11}$ Imaging may show reversible areas of oedema, mimicking ischaemia, although some patients eventually develop permanent deficits. The diagnosis requires a history of recurrent episodes or a history of similar attacks in other family members (familial hemiplegic migraine). There is an association with MELAS and cerebral autosomal dominant arteriopathy with subcortial infarcts and leukoencephalopathy (CADASIL). In practice, in almost all patients with hemiplegia associated with a migraine-like headache, the apparent migraine-like headache is secondary to the ischaemia, rather than the other way round. Headache of varying severity is surprisingly common in acute ischaemic stroke and may be generalised, ipsilateral or contralateral to the infarct.

Migraineous infarction should only be accepted as the diagnosis if the patient has a prior history of typical migraine with aura and is left with a persistent focal deficit after otherwise recovering from their usual migraine aura. There is some evidence that a history of migraine with aura is a risk factor for ischaemic stroke, but the mechanism and strength of the association is uncertain. ${ }^{11}$ If the focal deficit is anatomically located outside the territory of the patient's usual aura, I am reluctant to accept the diagnosis of migraineous infarction and search hard for alternative explanations.

Migraine status may cause confusion. In this condition, the patient experiences a prolonged migraine aura, usually hemianopic, with or without other features of migraine. The focal deficit characteristically fluctuates in severity, coming and going over the course of a week or two, before full recovery. Focal deficits may also fluctuate in patients with spasm secondary to subarachnoid haemorrhage and cause diagnostic difficulty in patients in whom the headache of subarachnoid haemorrhage is unusually mild or not elicited because of confusion or dysphasia.

\section{Moyamoya}

Moyamoya was originally thought to be confined to the Asian population, but is occasionally seen in other races. ${ }^{12}$ It can also develop in children with sickle cell disease. The diagnosis is based on the finding of severe stenosis or occlusion of the terminal carotid artery and origins of the branches of the circle of Willis. The term refers to the

Additional investigations performed in young or
recurrent stroke (routine investigations are listed by
Bamford, page i3)
- Magnetic resonance imaging (MRI)
- Autoantibody screen
- Anticardiolipin antibodies
- Thrombophilia screen
- Sickle screen
- Serum homocysteine
- Serum lactate
- Syphilis serology
- Drug screen
- 24 hour electrocardiogram (ECG) recording
- Transthoracic echocardiography
- Transoesophageal echocardiography
- Cerebral angiography


appearances of fine collaterals on angiography, which appear during dynamic angiography like a hazy puff of smoke (moyamoya in Japanese). The diagnosis can be suspected from cross sectional MRI, which may show the dilated collaterals as flow voids in the basal ganglia and confirmed by vascular imaging (magnetic resonance angiography (MRA) or angiography). Management is difficult because of the desire to prevent both infarction and haemorrhage. I prescribe antihypertensive treatment to maintain as low a blood pressure as can be tolerated by the patient, and avoid anticoagulation and antiplatelet treatment. Extracranial to intracranial bypass surgery may have a role in patients with progressive ischaemia.

\section{Vasculitis}

Most patients presenting with stroke secondary to vasculitis have symptoms of systemic disease. Headache is a characteristic feature. Isolated cerebral vasculitis without other systemic features of vasculitis (primary angiitis of the central nervous system) is very rare, but should be suspected in the patient with recurrent stroke-like events and deteriorating intellectual function. ${ }^{13}$ Radiology is usually unhelpful in the diagnosis of vasculitis, but CT or MRI may show progressive deep white matter ischaemia and angiography occasionally shows beading. Cerebral biopsy is necessary to make the diagnosis and exclude other causes. Intensive immunosuppression with cyclophosphamide is often required to control vasculitis. A "trial of steroids" before cerebral biopsy may only partially control the vasculitis, while suppressing diagnostic features on biopsy, so is to be avoided.

\section{Takayasu's arteritis}

Takayasu's arteritis is an idiopathic granulomatous aortitis which leads to occlusion of the origins of the great vessels, most commonly the carotid arteries. ${ }^{14}$ The disease is usually seen in young women born in Asia, who present with TIA or stroke, but can occur in whites. There is often malaise and fever, and the erythrocyte sedimentation rate is raised without evidence of autoantibodies. Reduced amplitude or absence of one or both brachial pulses, with pronounced asymmetry of blood pressure readings between the upper and lower limbs, should suggest the diagnosis. Ultrasound may show thickening of the walls of the common carotid artery. Angiography is required to make the diagnosis. Prednisolone is usually sufficient to control the malaise and prevent progression of the disease. In some cases, bypass graft surgery may be necessary.

\section{Systemic lupus erythematosus}

Less than $5 \%$ of cases with cerebral lupus present with stroke as their first manifestation of systemic lupus erythematosus (SLE); in most of these cases there are systemic features to suggest the diagnosis - for example, alopecia, skin rash, nail fold or retinal infarcts. ${ }^{15}$ These should be looked for in all patients with stroke of uncertain cause, especially as SLE can also affect the elderly and the diagnosis can be overlooked. Serological tests for DNA binding are usually positive, but the titres may not match the severity of the disease and stroke can occur with normal values. A number of different mechanisms can be responsible for stroke in SLE, including coagulopathy associated with the lupus anticoagulant, small vessel vasculitis, cardiac embolism from Libman-Sacks endocarditis, and hypertension from renal involvement.

\section{Antiphospholipid antibody syndrome}

In the full syndrome, antiphospholipid antibodies are associated with recurrent venous and arterial thrombosis, thrombocytopenia, and recurrent miscarriages in younger women. ${ }^{16}$ There is a close association with SLE, but not all patients with the antiphospholipid antibody syndrome have serological or clinical features of SLE and these patients are said to have primary antiphospholipid antibody syndrome. The lupus anticoagulant is itself an antiphospholipid antibody. Anticardiolipin antibody is the most common antiphospholipid antibody measured in the laboratory. The significance of anticardiolipin antibodies as a single indicator of stroke risk in the absence of features of the full syndrome is uncertain. In patients with stroke and features of the full syndrome, especially if there is evidence of SLE, the diagnosis can be accepted as likely to be relevant to the cause of the stroke, although whether anticardiolipin antibodies have a pathogenic role or are markers of an autoimmune hypercoagulable state remains uncertain. In the majority of cases of stroke in whom anticardiolipin antibodies are detected without evidence of any other autoimmune disorder, the implications are unclear. Current evidence suggests that the finding of anticardiolipin antibodies does not necessarily confer a greater risk of recurrence than in patients without the antibody, other risk factors being similar. My current practice is therefore to treat patients with isolated anticardiolipin antibodies and stroke with antiplatelet treatment in the first instance. I only consider anticoagulation in patients with the full syndrome, active SLE, evidence of a major disturbance of coagulation in the laboratory indicated by a positive lupus anticoagulant test, or recurrent thrombotic events.

\section{Sneddon's syndrome}

Sneddon described the association of stroke with florid livedo reticularis in the absence of any other systemic disease. ${ }^{17}$ The condition is mainly seen in young women. Livedo reticularis is associated with a number of connective tissue disorders, including primary antiphospholipid antibody syndrome, but to make a diagnosis of Sneddon's syndrome the livedo reticularis should be florid and generalised and there should be no evidence of any other relevant disorder. Skin biopsy shows a non-vasculitic arteriopathy with thickening of the blood vessel walls. Some patients experience recurrent stroke, progressing to vascular dementia. Treatment appears ineffective, but a trial of anticoagulation would seem justified in patients with recurrent events, although cerebral haemorrhage has been described.

\section{Susac's syndrome (retinocochleocerebral vasculopathy)}

This rare condition is an idiopathic small vessel vasculopathy with a predilection for the retina, cochlea, and brain stem. ${ }^{18}$ Patients develop recurrent vascular episodes over a period of several years with focal visual loss, secondary to discrete small retinal infarcts, sudden unilateral deafness, tinnitus, and brain stem stroke. Cortical involvement is less common, but can lead to dementia. The combination of sudden visual loss, asymmetrical segmental visual field defects, and deafness should suggest the diagnosis. No treatment appears to be effective.

\section{Thrombophilia}

The term thrombophilia is used to describe an increased tendency to clinical thrombosis associated with laboratory evidence of an abnormality in the coagulation pathway, particularly deficiency of one of the natural anticoagulant factors (antithrombin III, protein C, protein S, heparin cofactor 2, and the factor $\mathrm{V}$ Leiden mutation). Inherited thrombophilia, particularly the factor $\mathrm{V}$ Leiden mutation, accounts for at least $20 \%$ of cases of venous thromboembolism 
in patients under the age of 45 years, and there is a clear association with cerebral venous thrombosis. ${ }^{19}$ However, most recent case-control studies found no association between thrombophilia and arterial stroke in the young. It is important to recognise that inherited heterozygote deficiency in one of the anticoagulant factors is found in a significant proportion of the normal population, often without any history of thrombosis, and may therefore be a coincidental finding in a patient with stroke. In addition, concentrations of protein $\mathrm{C}$, protein S, and antithrombin III are depressed after stroke. Blood tests should therefore be repeated at least three months after the acute event before concluding that inherited deficiency is present. If the patient has been anticoagulated, interpretation of the result may be difficult because warfarin lowers the concentration of protein $\mathrm{C}$ and $\mathrm{S}$.

In general, it seems reasonable to ignore the association if other strong risk factors for stroke are found, but in patients in whom no obvious other cause is established life long anticoagulation should be considered. In some patients, particularly those with the factor $\mathrm{V}$ Leiden mutation, it may be more appropriate to anticoagulate only patients with recurrent thrombosis.

\section{Patent foramen ovale}

The relevance and optimum management of a patent foramen ovale in a patient with otherwise unexplained stroke is uncertain. Patent foramen ovale occurs in as many as 20 $40 \%$ of adults and therefore is likely to be a coincidental finding in many patients with stroke. ${ }^{20}$ For paradoxical embolism to occur, three coincidental conditions are required. Firstly, there must be a blood clot in the deep veins of the legs or pelvis which embolises to the right side of the heart. Secondly, there must be a communication of sufficient size to allow the blood clot through the atria. Thirdly, the pressure in the right atrium must be higher than in the left atrium. Normally the pressure is higher on the left side than on the right side, so even with patent foramen ovale the flow of blood is from left to right. However, the pressure may be increased on the right side of the heart to cause reversed flow from right to left if the patient also has right ventricular hypertrophy. Reversed flow can also occur transiently in patients with otherwise normal hearts by manoeuvres that raise intrathoracic pressure, such as straining at stool. Because all these features have to coincide, paradoxical embolism is probably a rare cause of stroke and I only accept the diagnosis as likely if the stroke occurs in association with prior deep vein thrombosis, pulmonary embolism or pulmonary hypertension. Given the doubts about the mechanism of stroke in most cases, I usually treat the patient with aspirin and only consider warfarin or percutaneous closure of the septal defect in patients with recurrent symptoms despite antiplatelet treatment.

\section{Combination of haemorrhage and infarction}

\section{Haemorrhagic transformation}

A number of disorders are associated with the combination of haemorrhage and infarction. The most common encountered in acute stroke is haemorrhagic transformation of an initially ischaemic infarct. Frank haemorrhage in this situation is often the result of spontaneous reperfusion of the infarct or therapeutic thrombolysis, and therefore is more likely to occur within the first day after onset of ischaemic stroke. The CT and MR appearances can be indistinguishable from a primary intracerebral haemorrhage, and it is only the existence of a previous scan that allows the diagnosis of secondary haemorrhage to be established. At a week after stroke onset, petechial haemorrhage into the infarct is very common and is visible on MRI in as many as $80 \%$ of patients with large middle cerebral artery territory infarcts, particularly in the putamen. My own practice is to stop or delay starting antiplatelet treatment or anticoagulation for 2-4 weeks in those with haemorrhagic transformation, unless the bleeding is only petechial, but thereafter I ignore the haemorrhage in making decisions about appropriate prevention of recurrence.

Cerebral venous thrombosis commonly causes both infarction and haemorrhage. This is discussed elsewhere in this supplement.

\section{Microhaemorrhage}

The recent introduction of $\mathrm{T} 2{ }^{\star} \mathrm{MR}$ sequences as a routine investigation in our unit for stroke and TIA has led to the new dilemma of how we should manage patients with microhaemorrhages, which may be detected on MR sequences in the basal ganglia of patients presenting with apparent lacunar infarction. Usually the patient has clear evidence of ischaemic small vessel disease in addition to the microhaemorrhages, and it is unclear whether the symptoms have resulted from the haemorrhages or from lacunar infarction. In patients with florid changes, I have taken the view that anticoagulation and aspirin are both contraindicated.

\section{Intravascular lymphoma}

Intravascular lymphoma (malignant angioendotheliosis) is an important differential diagnosis of the combination of haemorrhage and infarction in separate locations. This is a rare malignancy in which neoplastic lymphoid cells proliferate within capillaries, small veins, and arteries with little or no adjacent parenchymal involvement. ${ }^{21}$ The history is usually of recurrent stroke-like episodes, with either or both haemorrhage and infarction on imaging. Dementia, a spinal cord syndrome, and peripheral or cranial neuropathies have been described. It can produce an identical picture to primary angiitis of the central nervous system and distinction may only be possible on brain biopsy or postmortem. This disorder should be suspected if a patient has recurrent stroke-like episodes associated with white matter disease on the MRI. Often there is a haemorrhagic component to the stroke. Other causes of small vessel vasculitis may also occasionally cause both haemorrhage and infarction.

\section{Recurrent ischaemic stroke}

There is nothing more disappointing than the occurrence of recurrent stroke in patients who have taken every precaution to reduce their vascular risk and who have been receiving antiplatelet treatment. Unfortunately, aspirin on average only reduces the relative risk of stroke by about $13 \%$ and the addition of dipyridamole only approximately doubles the benefit, while at best clopidogrel confers an additional $8 \%$ benefit. ${ }^{22}$ Hence, recurrence is not unexpected, given that at least two thirds of patients do not appear to benefit from even combination antiplatelet treatment. What further should be done in patients with recurrent stroke or TIA, who have had optimum antiplatelet treatment, other than reiterating advice about vascular risk factors? Firstly, one should review the investigations and consider extending the range of investigations to include those normally performed only routinely in young stroke. Carotid or vertebral angiography should be considered to exclude high cervical or intracranial lesions, including atheromatous stenosis, and bifurcation stenoses missed by ultrasound in patients with carotid territory ischaemia. In this context, it should be 


\section{Summary 2}

- Cerebral biopsy is necessary to make the diagnosis of isolated cerebral vasculitis

- The significance of isolated anticardiolipin antibodies is uncertain

- Thrombophilia is strongly associated with cerebral venous thrombosis, but not arterial stroke

- Patent foramen ovale may be a coincidental finding in many patients with stroke

- Anticoagulation for ischaemic stroke prevention may be harmful unless a clear cardiac cause has been established

- In patients with hypertension, a target blood pressure of $140 / 85 \mathrm{~mm} \mathrm{Hg}$ or less should be set

remembered that one posterior cerebral artery is supplied by the internal carotid artery via a dominant posterior communicating artery in about $5 \%$ of the population. Thus, occipital infarction may sometimes result from carotid stenosis. Until recently, I considered conventional catheter angiography to be justified in patients with recurrent symptoms, but it is becoming clear that good quality extracranial and intracranial MRA, especially if contrast is used, can obviate the need for catheter angiography in many cases. The advantages of adequate angiography is that logical decisions can be made about treatment; in some cases of recurrent symptoms resistant to medical treatment, stenting of carotid or vertebral stenosis may be appropriate, even if the lesion is not amenable to surgery.

The only situation where anticoagulation has been shown to be beneficial in preventing recurrent stroke is in patients with atrial fibrillation. ${ }^{23}$ In contrast, the only large randomised trial of warfarin treatment for stroke prevention after presumed atherothrombotic stroke or TIA was stopped early because of an excess of cerebral haemorrhage in the warfarin group. ${ }^{24}$ The latter was more common in patients over the age of 75 years, in patients with severe leukoaraiosis on $\mathrm{CT}$, and in those with higher international normalised ratio (INR) values. Therefore, I do not recommend anticoagulation in patients with recurrent symptoms despite optimum antiplatelet treatment, unless investigations have shown a clear cardiac source of embolism, or confirmed severe $(>70 \%)$ atheromatous disease not amenable to surgery or stenting, and there are no contraindications to anticoagulation. Instead, I concentrate on optimising medical treatment in addition to antiplatelet treatment. Control of hypertension can often be improved and a target blood pressure of 140/85 $\mathrm{mm} \mathrm{Hg}$ or less should be set, informing both the patient and his or her general practitioner about the desirable target. Similarly, I encourage as good as possible control of hyperglycaemia in diabetics. There is good evidence that statins reduce both myocardial infarction and stroke in patients with ischaemic heart disease, ${ }^{25}$ and it is very likely that similar benefits will be shown in the ongoing trials of statin treatment in stroke. I therefore recommend statin treatment to most patients with recurrent symptoms unless their cholesterol is below $5 \mathrm{mmol} / \mathrm{l}$.

\section{References}

1 Blight A, Pereira AC, Brown MM. A single consultation cerebrovascular clinic is cost effective in the management of transient ischaemic attack and minor stroke. J $R$ Coll Physicians Lond 2000;34:452-5.

2 Russell RW, Page NG. Critical perfusion of brain and retina. Brain 1983;106:419-34.

3 Fisher CM. Late-life migraine accompaniments-further experience. Stroke 1986;17:1033-42.

4 Hodges JR, Warlow CP. Syndromes of transient amnesia: towards a classification. A study of 153 cases. J Neurol Neurosurg Psychiatry 1990;53:834-43.

5 Schwab S, Steiner T, Aschoff A, et al. Early hemicraniectomy in patients with complete middle cerebral artery infarction. Stroke 1998;29:1888-93.

6 Duke RJ, Bloch RF, Turpie AG, et al. Intravenous heparin for the prevention of stroke progression in acute partial stable stroke. Ann Intern Med 1986;105:825-8.

7 Haley Jr EC, Kassell NF, Torner JC. Failure of heparin to prevent progression in progressing ischaemic infarction. Stroke 1988;19:10-14.

8 Pereira AC, Brown MM. Aspirin or heparin in acute stroke. Br Med Bull 2000;56:413-21.

- Anticoagulation treatment for acute ischaemic stroke is reviewed in this volume of the British Medical Bulletin, which contains many other up to date reviews on stroke treatment and is highly recommended.

9 Carolei A, Marini C, Ferranti E, et al. A prospective study of cerebral ischaemia in the young. Analysis of pathogenic determinants. The National Research Council study group. Stroke 1993;24:362-7.

10 Ciafaloni E, Ricci E, Shanske S, et al. MELAS: clinical features, biochemistry, and molecular genetics. Ann Neurol 1992;31:391-8.

11 Welch KMA, Tatemichi TK, Mohr JP. Migraine and stroke. In: Barnett HJM, Mohr JP, Stein BM, et al, eds. Stroke: pathophysiology, diagnosis and management, 3rd ed. Philadelphia: Churchill Livingstone, 1998: 845-67.

- A comprehensive review of the whole problem of migraine and stroke in one of the major textbooks of stroke.

12 Suzuki J, Kodama N. Moyamoya disease-a review. Stroke 1983;14:104-9.

13 Ferro JM. Vasculitis of the central nervous system. J Neurol 1998;245:766-76.

14 Hall S, Buchbinder R. Takayasu's arteritis. Rheum Dis Clin North Am 1990;16:411-22.

15 Brown MM, Swash M. Systemic lupus erythematosus. In: Vinken PJ, Bruyn GW, Klawans HL, eds. Handbook of clinical neurology, revised series, vascular diseases part III, vol 55. Amsterdam: Elsevier Science Publishers, 1989:369-85.

16 Montalban J, Codina A, Ordi J, et al. Antiphospholipid antibodies in cerebral ischemia. Stroke 1991;22:750-3.

17 Tourbah A, Piette JC, Iba-Zizen MT, et al. The natural course of cerebral lesions in Sneddon syndrome. Arch Neurol 1997;54:53-60.

18 Susac JO. Susac's syndrome: the triad of microangiopathy of the brain and retina with hearing loss in young women. Neurology 1994:44:591-3.

19 Martinelli I, Landi G, Merati G, et al. Factor V gene mutation is a risk factor for cerebral venous thrombosis. Thromb Haemost 1996;75:393-4

20 Autore C, Cartoni D, Piccininno M. Multiplane transesophageal echocardiography and stroke Am J Cardiol 1998;81:79G-81G.

21 Glass J, Hochberg FH, Miller DC. Intravascular lymphomatosis. A systemic disease with neurologic manifestations. Cancer 1993;71:3156-64.

22 McCabe DJH, Brown MM. Prevention of ischaemic stroke-antiplatelets. Br Med Bull 2000;56:510-25.

23 EAFT (European Atrial Fibrillation Trial) Study Group. Secondary prevention in non-rheumatic atrial fibrillation after transient ischaemic attack or minor stroke. Lancet 1993;342:1255-62.

- This study convincingly established the benefits of anticoagulation after stroke and TIA in patients with atrial fibrillation.

24 The Stroke Prevention in Reversible Ischaemia Trial (SPIRIT) Study Group. A randomised trial of anticoagulants versus aspirin after cerebral ischaemia of presumed arterial origin. Ann Neurol 1997;42:857-65.

25 Hess DC, Demchuk AM, Brass LM, et al. HMG-CoA reductase inhibitors (statins): a promising approach to stroke prevention. Neurology 2000:54:790-6. 